\title{
Diffractive production of isolated photons at HERA
}

\section{Peter Bussey*}

School of Physics and Astronomy

University of Glasgow

Glasgow, United Kingdom, G12 8QQ. E-mail: peter.bussey@glasgow. ac.uk

For the ZEUS Collaboration.

The ZEUS detector at HERA has been used to measure the photoproduction of isolated photons in diffractive events. Cross sections are evaluated in the photon transverse-energy and pseudorapidity ranges $5<E_{T}^{\gamma}<15 \mathrm{GeV}$ and $-0.7<\eta^{\gamma}<0.9$, inclusively and with a jet with transverse energy and pseudorapidity in the ranges $4<E_{T}^{\text {jet }}<35 \mathrm{GeV}$ and $-1.5<\eta^{\text {jet }}<1.8$, for an integrated luminosity of $374 \mathrm{pb}^{-1}$. Further kinematic variables studied include the fractions of the incoming photon energy and of the colourless exchange ("Pomeron") energy that are imparted to a photon-jet final state. Comparison is made to predictions from the RAPGAP Monte Carlo simulation.

XXIII International Workshop on Deep-Inelastic Scattering,

27 April - May 12015

Dallas, Texas

${ }^{*}$ Speaker. 


\section{Introduction}

In diffractive hadronic particle interactions, a colour-neutral object is exchanged, frequently referred to as the "pomeron". Diffractive scattering off protons may be initiated by a second incoming hadron, or even by a real or virtual photon. At the HERA ep collider, diffractive processes have been studied both in photoproduction and in deep inelastic scattering, the photoproduction processes consisting of those in which the exchanged virtual photon is quasi-real. The diffractive process is characterised by a forward nucleon, followed by a "gap" in rapidity in which little or no energetic scattering is found until the central region of the process where the hard final state is detected and measured.

This talk presents measurements in which a hard isolated "prompt" photon is detected in the central region of the ZEUS detector and may be accompanied by a jet. Such processes, while rare, are interesting for several reasons. An outgoing photon must be radiated from a charged partonic object, namely a quark, and therefore demonstrates the presence of a quark content in the pomeron or of higher-order processes in which both the pomeron and incident photon couple to quarks. In general, they allow QCD-based models to be tested in order to improve our understanding of a type of process which is important at high energies.

The observed processes also include "fragmentation processes" in which a photon is radiated within a jet. Such processes are suppressed by requiring that the outgoing photon be isolated. The present measurements follow an earlier study by H1 [1] of inclusive diffractive high energy prompt photons as a function of transverse momentum. Analyses of isolated photons in non-diffractive photoproduction have also been made by the ZEUS and H1 collaborations [2, 3].

\section{Kinematic quantities}

In "direct" photoproduction processes, the entire incoming photon is absorbed by an outgoing quark from the incoming proton, while in "resolved" processes, the photon's hadronic structure provides a quark or gluon that interacts with a parton from the proton. These two classes of process (fig. 1), which are unambiguously defined only at lowest order, may be partially distinguished in
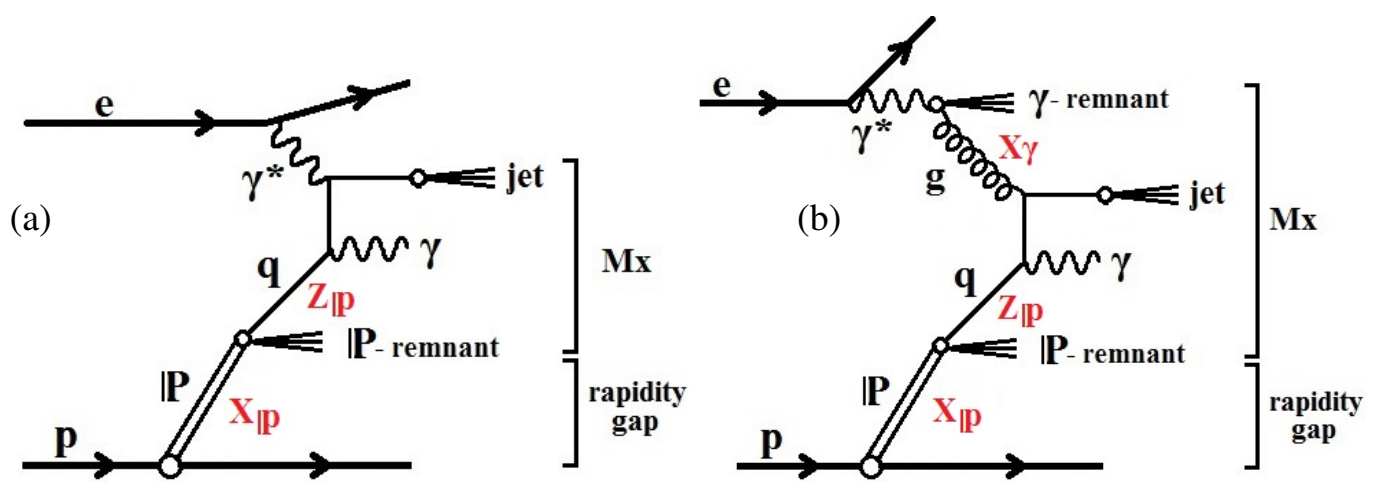

Figure 1: Examples of the diffractive production of a prompt photon and a jet in $e p$ scattering from (a) direct (b) resolved photons. 
events containing a high- $E_{T}$ photon and a jet by means of the quantity

$$
x_{\gamma}^{\text {meas }}=\frac{E^{\gamma}+E^{\mathrm{jet}}-p_{Z}^{\gamma}-p_{Z}^{\text {jet }}}{E^{\text {all }}-p_{Z}^{\text {all }}},
$$

which measures the fraction of the incoming photon energy that is given to the photon and the jet. Similarly, direct and resolved pomeron processes may be defined. The quantities $E^{\gamma}$ and $E^{\text {jet }}$ denote the energies of the photon and the jet, respectively, $p_{Z}$ denotes the corresponding longitudinal momenta, and the suffix "all" refers to all the final-state particles or detector-measured objects in an event. The presence of direct processes generates a prominent peak in the cross section at high $x_{\gamma}^{\text {meas }}$ values.

When the proton, with energy $E_{p}$, radiates an object such as a pomeron, which interacts with all or part of an incoming photon, the fraction of the proton energy taken by the radiated pomeron is given to a good approximation by:

$$
x_{\mathbb{P}}=\left(E^{\text {all }}+p_{Z}^{\text {all }}\right) / 2 E_{p} .
$$

The fraction of the pomeron energy that takes part in the hard interaction that generates the outgoing photon and jet is given to a good approximation by:

$$
z_{\mathbb{P}}^{\text {meas }}=\frac{E^{\gamma}+E^{\mathrm{jet}}+p_{Z}^{\gamma}+p_{Z}^{\text {jet }}}{E^{\text {all }}+p_{Z}^{\text {all }}} .
$$

\section{Experimental method}

The measurements are based on two data samples corresponding an integrated luminosities of 91 and $374 \mathrm{pb}^{-1}$, taken during the years 1998-2000 and 2004-2007 respectively with the ZEUS detector at HERA, and referred to as HERA-I and HERA-II samples respectively. During these periods, HERA ran with an electron or positron beam energy of $27.5 \mathrm{GeV}$ and a proton beam energy of $E_{p}=920 \mathrm{GeV}$. The samples include $e^{+} p$ and $e^{-} p$ data ${ }^{1}$.

A detailed description of the ZEUS detector is given elsewhere [4]. Charged particles were measured in the central tracking detector and a silicon micro vertex detector which operated in a magnetic field of $1.43 \mathrm{~T}$ provided by a thin superconducting solenoid. The barrel electromagnetic calorimeter (BEMC) cells had a pointing geometry aimed at the nominal interaction point. Its fine granularity allows the use of shower-shape distributions to distinguish isolated photons from the products of neutral meson decays such as $\pi^{0} \rightarrow \gamma \gamma$.

Monte Carlo (MC) event samples were employed to evaluate the detector acceptance and event-reconstruction efficiency, and to provide signal and background distributions. The program RAPGAP 3.2 [5] was used to generate the diffractive process $p e \rightarrow p e \gamma X$ for direct and resolved incoming virtual photon exchange, where $X$ denotes any other particles. The diffractive proton pdf set H1-B (2006) was used, and for the resolved photon the pdf set SaSG 1D LO. The program Pythia 6.416 [6] was used to generate direct and resolved prompt-photon photoproduction processes for background calculations, and also $2 \rightarrow 2$ parton-parton scattering processes not involving

\footnotetext{
${ }^{1}$ Hereafter "electron" refers to both electrons and positrons unless otherwise stated.
} 
photons ("dijet events"), making use of the CTEQ4 and GRV proton and photon parton densities. The program was run using the default parameters with minor modifications. Non-diffractive photoproduction event samples were also generated using the HERWIG 6.510 program [7], again with minor modifications to the default parameters. The PYTHIA and HERWIG programs differ significantly in their treatment of parton showers, and in the use of a string-based hadronisation scheme in Pythia but a cluster-based scheme in HeRwig.

The measured photons are accompanied by backgrounds from neutral mesons in hadronic jets, in particular $\pi^{0}$ and $\eta$, where the meson decay products create an energy cluster in the BCAL that passes the selection criteria for a photon. Although not generated diffractively, the dijet event samples enabled such background events to be distinguished in the analysis..

The basic event selection and reconstruction were performed as previously [2]. A three-level trigger system was used to select events online. The event analysis made use of energy-flow objects (EFO's), constructed from clusters of calorimeter cells, associated with tracks when appropriate, and also unassociated tracks. Photon candidates were identified as EFO's with no associated track, and with at least $90 \%$ of the reconstructed energy measured in the BEMC.

Jets were reconstructed using all the EFO's in the event, including photon candidates, by means of the $k_{T}$ clustering algorithm in the $E$-scheme in the longitudinally invariant inclusive mode with the radius parameter set to 1.0. To reduce the fragmentation contribution and the background from the decay of neutral mesons within jets, the photon candidate was required to be isolated from other hadronic activity. This was imposed by requiring that the photon-candidate EFO had at least $90 \%$ of the total energy of the reconstructed jet of which it formed a part.

Events were finally selected with the following kinematic conditions: Each event was required to contain a photon candidate with a reconstructed transverse energy, $E_{T}^{\gamma}$, in the range $5<E_{T}^{\gamma}<15 \mathrm{GeV}$ and with pseudorapidity, $\eta^{\gamma}$, in the range $-0.7<\eta^{\gamma}<0.9$; The hadronic jet, when used, was required to have $E_{T}^{\text {jet }}$ between 4 and $35 \mathrm{GeV}$ and to lie within the pseudorapidity, $\eta^{\text {jet }}$, range $-1.5<\eta^{\text {jet }}<1.8$; To select diffractive events further conditions were applied, the first of which was that the maximum pseudorapdity for EFO's with energy above $0.4 \mathrm{GeV}$ satisfied $\eta_{\max }<2.5$. A second diffractive condition was that $x_{\mathbb{P}}<0.03$. For the HERA-I data sample, the energy in the Forward Plug Calorimeter was required to be less than $1 \mathrm{GeV}$. This calorimeter was not present in the HERA-II running.

The HERA-II data sample was used in the main analysis described here. The HERA-I data were analysed similarly, with the addition of the selection on the Forward Plug Calorimeter, and were used to provide a correction as described below. A further background arises from Deeply Virtual Compton Scattering events, which were removed from the sample.

The selected samples include background events arising from neutral meson decays. The photon signal was extracted statistically following the approach taken in previous ZEUS analyses, and used the energy-weighted width measured in the $Z$ direction, $\langle d Z\rangle$, of the BEMC energy cluster that comprised the photon candidate. For each measured cross-section bin, the number of isolatedphoton events in the data was determined by a Maximum-Likelihood fit to the $\langle\delta Z\rangle$ distribution in the range $0.05<\langle\delta Z\rangle<0.8$, varying the relative fractions of the signal and background components as represented by histogram templates obtained from the MC (fig. 2(a)).

After applying the selections described above, the distribution of the events in $x_{\gamma}^{\text {meas }}$ is as shown in fig. 2(b). A 80:20 mixture of direct:resolved RAPGAP events gives a reasonable descrip- 

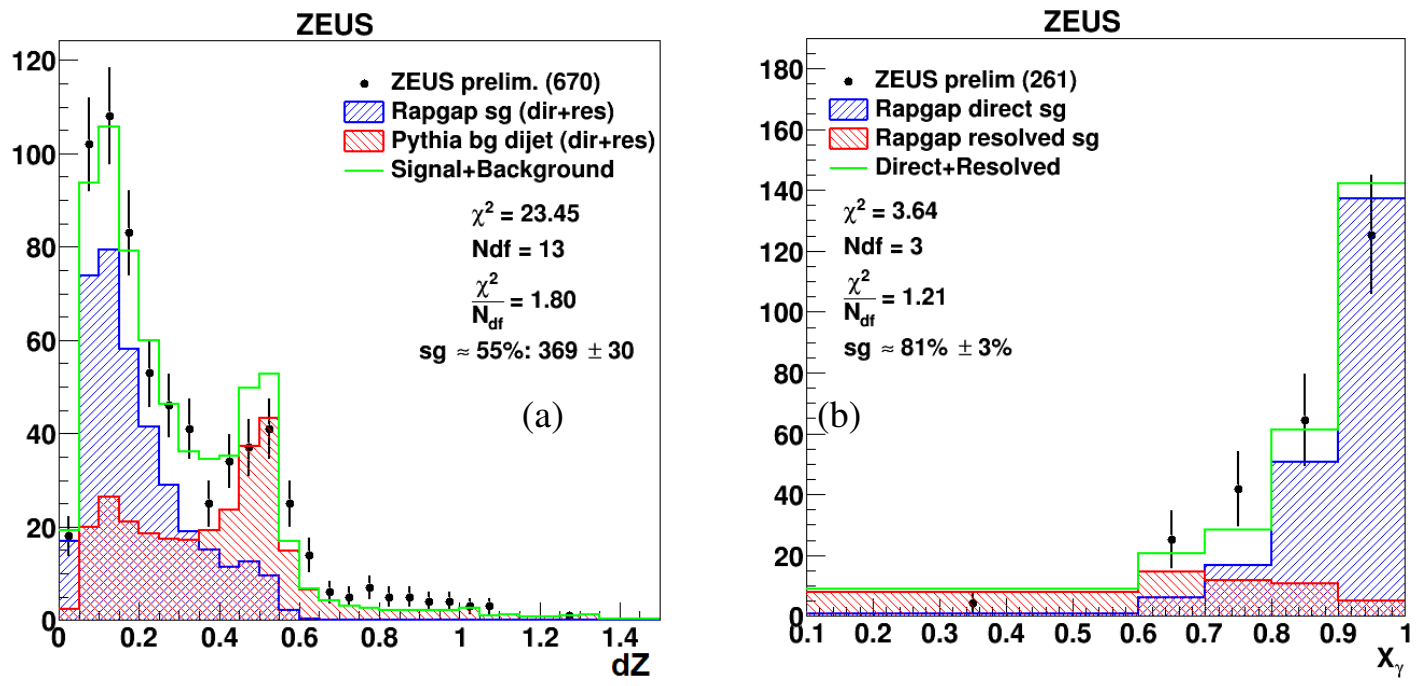

Figure 2: (a) Distribution of $\langle\delta Z\rangle$ for selected photon candidates. (b) Distribution of photon events, after experimental selections, as a function of $x_{\gamma}^{\text {meas }}$ per unit interval of 0.1 in $x_{\gamma}^{\text {meas }}$, compared to a mixture of reweighted RAPGAP-generated direct and resolved events using the model described in the text.

tion of the data and was used subsequently. To evaluate the acceptances, allowance must be made for the different acceptances for the direct and the resolved processes. A weighting factor as a function of $\eta^{\max }$ was applied to give agreement between the RAPGAP prediction and the data; however this has only a small effect on the acceptance calculation. A bin-by-bin correction method was then used to determine the production cross section in a given variable.

Two further corrections were applied to the HERA-II data, which were used to provide the results presented below. The total cross sections for the diffractive processes were evaluated, and the HERA-II data were rescaled to have the same total cross section as measured using the HERA-I data. This compensated for a potential loss of events in the HERA-II data due to scattering of forward-generated particles off a beamline magnet that occupied most of the central aperture of the forward ZEUS calorimeter in the HERA-II running, but which was absent during the HERA-I running. The HERA-I Forward Plug Calorimeter removed additional non-diffractive events in this angular region.

A second correction was needed to remove non-diffractive events that succeeded in passing the diffractive event selections described above. It was evaluated using Pythia and HeRwig photoproduction event samples, which were normalised to the data sample after extracting the photon signal without applying the diffractive event selections. The diffractive event selections were then applied to the Monte Carlo samples to estimate the contribution of the photoproduction to the observed data. The mean of the two corrections was applied.

\section{Results}

Differential cross sections were calculated for the diffractive production of an isolated photon, inclusive and with at least one accompanying jet. The kinematic region was defined by $Q^{2}<$ 
$1 \mathrm{GeV}^{2}, 0.2<y<0.7,-0.7<\eta^{\gamma}<0.9,5<E_{T}^{\gamma}<15 \mathrm{GeV}, 4<E_{T}^{\text {jet }}<35 \mathrm{GeV}$ and $-1.5<\eta^{\text {jet }}<$ 1.8. The diffractive condition consisted in requirements that $x_{\mathbb{P}}<0.03$ and $\eta^{\max }<2.5$. These quantities were evaluated at the hadron level in the laboratory frame, matching the cuts used at the detector level, and the jets were formed according to the $k_{T}$ clustering algorithm with the radius parameter set to 1.0. Photon isolation was imposed such that at least $90 \%$ of the energy of the jet-like object containing the photon originated from the photon.

Results are shown in fig. 3, together with predictions from RAPGAP, normalised to the data. Cross sections in $E_{T}^{\text {jet }}$ above $15 \mathrm{GeV}$ are omitted from fig. 3(c) owing to limited statistics, but this kinematic region is included in the other cross-section measurements. The results here are inclusive of proton excitation processes which have been estimated to comprise approximately $16 \%$ of the total diffractive cross section.

The most significant sources of systematic uncertainty arose from the scaling of the HERAII cross sections to the HERA-I data, treated as a systematic uncertainty although its origin is statistical. This amounts to an uncertainty of $\pm 20 \%$. The next most significant source comes from the uncertainty in the non-diffractive correction and was typically $\pm 10 \%$. A further uncertainty can be estimated by using the HERWIG MC to model the signal and background events. Less important uncertainties arose from the calibrations of the photon and jet energy scales, the modelling of the resolved and direct ratios in the acceptance calculation and the modelling of the background. The effect of all of these was estimated by including an overall common scaling uncertainty of $\pm 25 \%$ on all the cross sections. The narrow uncertainty bars on the figures include this in combination with the statistical uncertainties, which are indicated by a thick bar.

The cross section for photons with a jet is seen to be a high fraction of the inclusive photon cross section. For the quantity $z_{\mathbb{P}}^{\text {meas }}$ the data show a peak at the upper end of the distribution which is not well described by RAPGAP. In all other cases, RAPGAP gives a good description of the data.

\section{References}

[1] H1 Collaboration, V. Andreev et al., Phys. Lett. B 672 (2009) 219

[2] ZEUS Collaboration, H. Abramowicz et al., Phys. Lett. B 730 (2014) 293

[3] ZEUS Collaboration, J. Breitweg et al., Phys. Lett. B 413 (1997) 201

ZEUS Collaboration, J. Breitweg et al., Phys. Lett. B 472 (2000) 175

ZEUS Collaboration, S. Chekanov et al., Phys. Lett. B 511 (2001) 19

ZEUS Collaboration, S. Chekanov et al., Eur. Phys. J. C 49 (2007) 511

H1 Collaboration, A. Aktas et al., Eur. Phys. J. C 38 (2004) 437

[4] ZEUS Collaboration, U. Holm (ed.), The ZEUS Detector. Status Report (unpublished), DESY (1993), available on http: / / www-zeus.desy.de/bluebook/bluebook.html

[5] H. Jung, http: //www . desy . de/ jung/rapgap.html

[6] T. Sjöstrand et al., JHEP 05 (2006) 26

[7] G. Corcella et al., JHEP 01 (2001) 010 
ZEUS

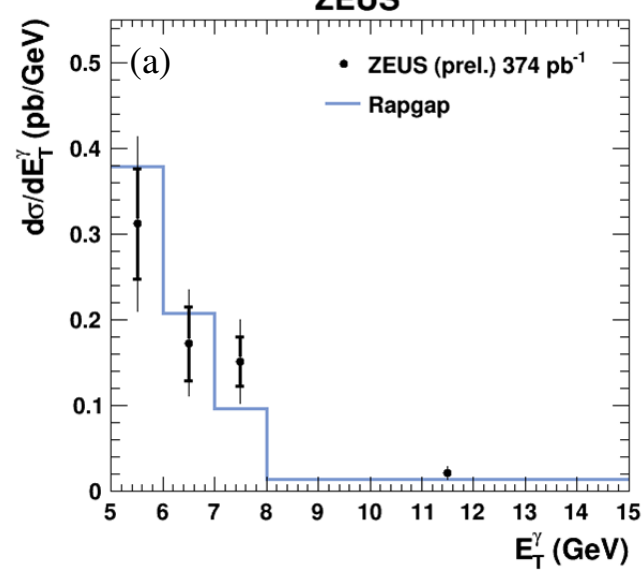

ZEUS

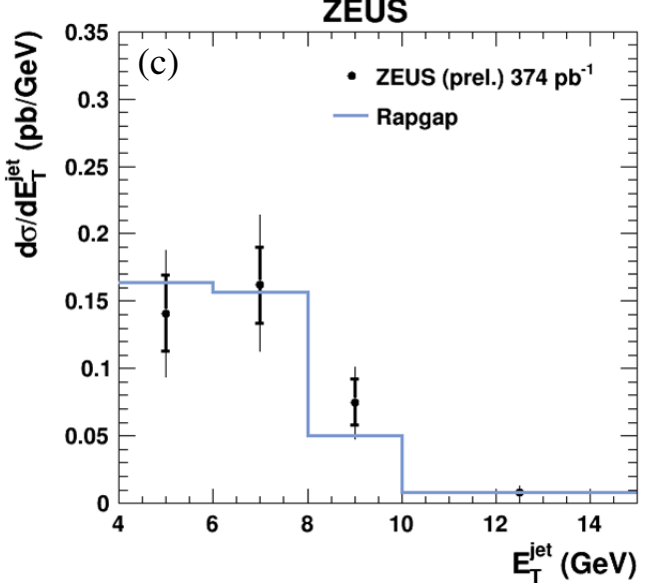

ZEUS

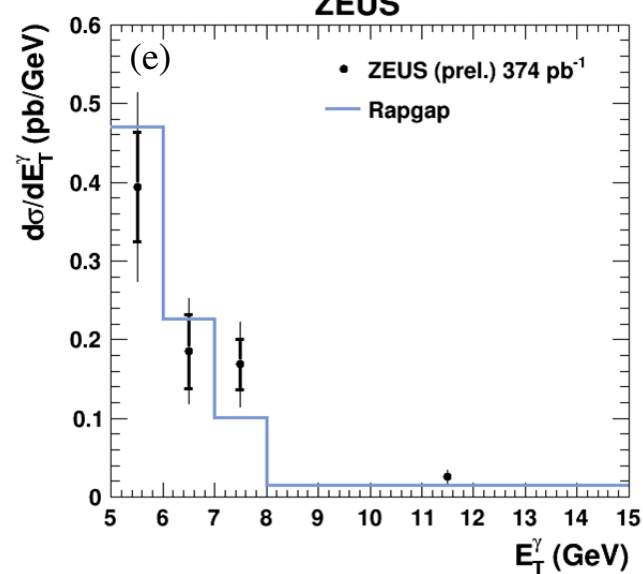

ZEUS

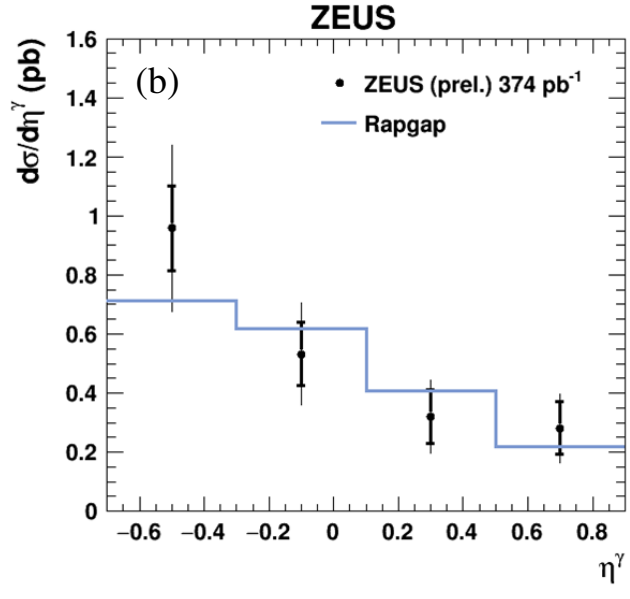

ZEUS

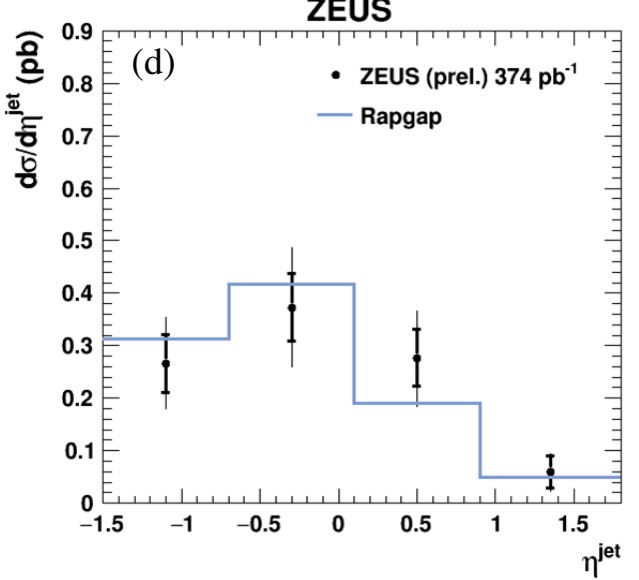

ZEUS

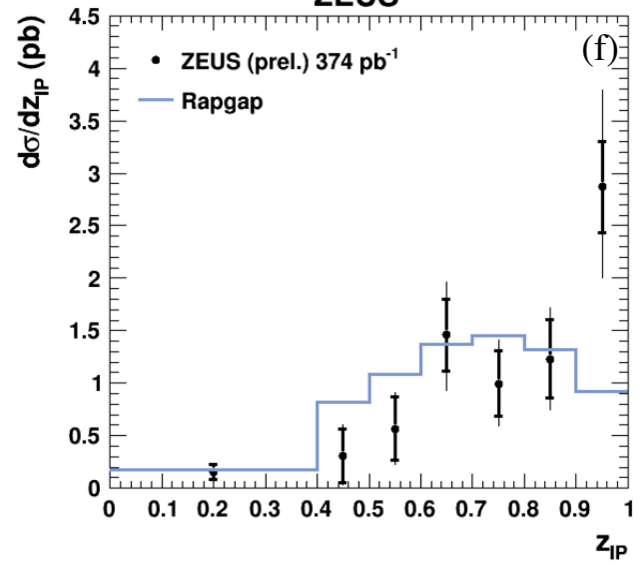

Figure 3: Differential cross sections as functions of (a) $E_{T}^{\gamma}$, (b) $\eta^{\gamma}$, (c) $E_{T}^{\text {jet }}$, and (d) $\eta^{\text {jet }}$, for events containing an isolated photon accompanied by a jet, (e) $E_{T}^{\gamma}$ for inclusive events containing an isolated photon, and (f) $z_{\mathbb{P}}^{\text {meas }}$ for events containing an isolated photon and a jet. Comparisons are made to normalised predictions from RAPGAP, evaluated without reweighting. 